Correspondencia a:

${ }^{1}$ Centro de investigación de fonoaudiología UNITEPC. "CIFU".

Cochabamba - Bolivia.

${ }^{2}$ Médico Cirujano. Especialista en Audiología en Universidad de Salamanca España. Médico responsable del Área de Audiología de la Corporación del Seguro Social Militar COSSMIL. Docente de Audiología UNITEPC. Cochabamba - Bolivia.

Email de contacto:

angelaord.009@gmail. com

cammy.direccion@gmail. com

Procedencia y arbitraje: No comisionado, sometido a arbitraje externo

Recibido para publicación: 28 de agosto del 2020 Aceptado para publicación: 15 de octubre del 2020

Citar como:

Ordoñez Luna AC, Lazarte Villarroel IT. Rehabilitación auditiva posterior a un implante coclear métodos tradicionales y método novedoso. Recisa UNITEPC. 2020;7(2):20-33.

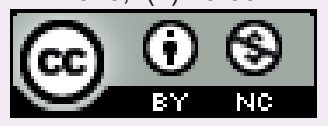

Esta obra está bajo una Licencia Creative Commons Atribución-NoComercial 4.0 Internacional.

\section{Rehabilitación auditiva posterior a un implante coclear métodos tradicionales y método novedoso}

\author{
Hearing rehabilitation after a cochlear implant; \\ traditional and new methods
}

\section{Reabilitação auditiva após implante coclear - métodos tradicionais e innovador}

\author{
Angela Carolina Ordoñez Luna ${ }^{1}$ \\ Ibeth Tatiana Lazarte Villarroel ${ }^{2}$
}

\section{Resumen}

Introducción: El implante coclear es un pequeño dispositivo biomédico electrónico, que se inserta a pacientes con hipoacusia sensorioneural profunda uni o bilateralmente, de origen coclear y que tiene como fin ayudar a escuchar, posterior al proceso de implantación. Existen métodos tradicionales que nos ayudan durante la rehabilitación auditiva. El objetivo de este trabajo es compilar información sobre los métodos tradicionales y novedosos dentro la rehabilitación auditiva posterior a un implante coclear. Metodología: Se realizó una búsqueda bibliográfica en las bases de datos electrónicas Elsevier, PubMed, NCBI y Google Académico. Desarrollo: se revisaron 458 títulos, de los cuales cumplían con nuestros criterios de selección 51 artículos. Los métodos tradicionales, así como la inserción de la musicoterapia como intervención novedosa tienen como fin darle calidad de vida a la persona con deficiencia auditiva. Discusión y conclusiones: Los hallazgos realizados en este trabajo ya se consideran evidencia científica y la finalidad es que sirvan como base para futuras investigaciones en nuestro país. Este tipo de cirugías cada vez serán de mayor demanda y requiere el conocimiento del tema por parte de nuestros estudiantes y profesionales especialistas del área.

Palabras claves: Implantes Cocleares, Pérdida Auditiva Sensorineural, Corrección de Deficiencia Auditiva, Métodos (Fuente: Descriptores en Ciencias de la Salud - DeCS).

\section{Summary}

Introduction: The cochlear implant is a small electronic biomedical device, which is inserted in patients with profound sensorineural hearing loss unilaterally or bilaterally, with cochlear origin and whose purpose is to help listening, after the implantation process. There are traditional methods that help us during hearing rehabilitation. This work's objective is to compile information about traditional and new methods in hearing rehabilitation after a cochlear implant. Methodology: A bibliographic search 
was carried out in the electronic databases Elsevier, PubMed, NCBI and Google Scholar. Development: 458 titles were reviewed, from which 51 articles fulfill our selection criteria. The traditional methods, as well as the music therapy insertion as a new intervention, have the aim to give life quality to the person with hearing impairment. Discussion and conclusions: The findings made in this work are already considered scientific evidence and the purpose is that they serve as a base for future research in our country. This type of surgery will be increasingly in demand and requires knowledge about the subject by our students and specialist professionals in the area.

Keywords: Cochlear Implants, Hearing Loss, Sensorineural, Correction of Hearing Impairment, Methods (Source: DeCS - Health Sciences Descriptors).

\section{Resumo}

Introdução: O implante coclear é um pequeno dispositivo eletrônico biomédico, que é inserido em pacientes com perda auditiva neurossensorial profunda, unilateral ou bilateralmente, de origem coclear; onde, tem por objetivo auxiliar na escuta, após o processo de implantação. Existem métodos tradicionais que nos auxiliam na reabilitação auditiva. O objetivo deste trabalho é compilar informações sobre os métodos tradicionais e inovadores de reabilitação auditiva após o implante coclear. Metodologia: Foi realizada uma busca bibliográfica nas bases de dados eletrônicas Elsevier, PubMed, NCBI e Google Scholar. Desenvolvimento: foram revisados 458 títulos, dos quais 51 artigos atenderam aos nossos critérios de seleção. Os métodos tradicionais, assim como a inserção da musicoterapia como uma nova intervenção, visam dar qualidade de vida à pessoa com deficiência auditiva. Discussão e conclusões: Os achados deste trabalho já são considerados evidências científicas e a finalidade é que sirvam de base para futuras pesquisas em nosso país. Este tipo de cirurgia será cada vez mais procurado e requer conhecimento do assunto por parte de nossos alunos e profissionais especializados na área.

Palavras chave: Implantes Cocleares, Neurossensorial, Correção de Deficiência Auditiva, Métodos (Fonte: DeCS - Descritores em Ciências da Saúde).

\section{Introducción}

La rehabilitación auditiva es el entrenamiento de la audición, por medio de distintos métodos que promueve la detección, discriminación, reconocimiento y comprensión de los estímulos sonoros, que tienen como objetivo desarrollar estas habilidades auditivas perdidas en la persona (1).

El implante coclear es un pequeño dispositivo biomédico electrónico, que se inserta por medio de una cirugía a pacientes prelocutivos o postlocutivos con hipoacusia sensorioneural profunda uni o bilateralmente, de origen coclear y que tiene como fin ayudar a escuchar (2)

En el año 2012 se registró alrededor de 324,200 personas en todo el mundo que habían recibido estos implantes y solo en Estados Unidos son unos 58,000 adultos y cerca de 38,000 niños $(3,4)$.

Posterior al proceso de la implantación, existen métodos tradicionales que nos ayudan durante la rehabilitación auditiva, los clasificamos en: métodos orales que abarca el auditivo verbal y el método verbo tonal, los gestuales ya sea por medio de la lengua de 
signos o dactilología y los métodos mixtos que incluye comunicación bimodal, palabra complementada y comunicación total. Un estudio realizado en Chile indica que dentro esta variedad de opciones, el recurso más utilizado es el método oral $(5,6)$.

Ahora bien, se están realizando distintos estudios de investigación donde se considera la musicoterapia como un método novedoso dentro la rehabilitación auditiva principalmente en la población infantil, puesto que les genera interés por la percepción de sonido, permitiéndole distinguir la estimulación de ritmo, melodía y timbre conforme se realice el avance en la terapia, el niño puede adquirir modelos de sonido. En un estudio en Colombia se demuestra que los niños que comprenden la edad de 8 y 10 años muestran una aproximación importante desde la musicoterapia hacia la imitación de los patrones melódicos vocálicos $(7,8)$.

De modo que, los métodos tradicionales, así como la inserción de la musicoterapia como intervención novedosa tiene como fin darle calidad de vida a la persona con deficiencia auditiva, no obstante, la incorporación de esta nueva herramienta debe seguir sujeta a investigaciones con el fin de conocer sus efectos desde el punto de vista de la práctica basada en la evidencia (9).

Ciertamente, los avances científicos han permitido progresar en el campo de la deficiencia auditiva a través de la creación del implante coclear, sin embargo, este no devuelve una audición normal y la naturaleza de los estímulos sonoros, presenta notables diferencias respecto a la estimulación acústica habitual, de manera que no basta con la intervención quirúrgica. Un estudio refleja que, a partir del año 2020, alrededor de 20.000 implantes se realizarán cada año y esto genera preocupación por la incidencia que muestran estas cifras (10).

El objetivo de este trabajo es compilar información del tema, sobre los métodos tradicionales y novedosos dentro la rehabilitación auditiva posterior a un implante coclear, con el fin de explorar las mejores técnicas de intervención terapéutica y este proceso bien realizado beneficiará al usuario a recuperar su máximo nivel de funcionalidad e independencia.

\section{Metodología}

Este trabajo fue realizado en la gestión 2020, el diseño de esta investigación es descriptivo, ya que nos limitamos a describir la información encontrada durante la revisión teórica. Según la secuencia temporal es transversal, porque los datos se recolectaron en un tiempo determinado. Por el inicio del estudio en relación con la cronología de los hechos es prospectivo, porque que los datos se generaron en el transcurso de la revisión.

Como unidades de información se utilizaron artículos científicos de una base de datos que son: Elsevier, PubMed, NCBI y Google Académico a través de la gama de sus revistas indexadas. La búsqueda se realizó por medio de palabras clave como ser: implante coclear, rehabilitación auditiva en implante coclear, musicoterapia, método auditivo verbal, método verbotonal y también palabras en inglés auditory oral therapy, cochlear implant y music therapy.

\section{Desarrollo}

La búsqueda en las bases de datos electrónicas arrojó 458 títulos, de los cuales 51 
estudios cumplían con nuestros criterios de selección.

El déficit auditivo representa sin duda una gran preocupación, las cifras de prevalencia son alarmantes. Aproximadamente hay 360 millones de personas afectadas, en otras palabras, el $5 \%$ de la población total en el mundo sufren algún grado de discapacidad auditiva, según la OMS (11). La incidencia de hipoacusia sensorioneural profunda en niños es de 1 nacido afectado por cada 1000 y en adultos mayores los datos son alrededor del $50 \%$ de este tipo de discapacidad a partir de los 75 años (12).

La relación entre hombres y mujeres que sufren alguna deficiencia auditiva es similar, sin embargo, estos datos podrían variar dependiendo su etiología, ya sea, por origen genético, factores de riesgo y ocupación laboral (13).

Respecto a la trascendencia de gran importancia a considerar es que hoy en día los jóvenes están expuestos a ruidos diarios por el uso de sus dispositivos musicales, asistencia a centros de recreación como ser discotecas y conciertos y esto a la larga desencadenara en una pérdida de audición, por lo cual se hace, más frecuente ver adultos en edades más tempranas acudir a consulta, situación que no se veía antes (14).

El Instituto Nacional de Seguridad y Salud Ocupacional (Niosh) de los EE:UU sobre esta situación indica que, "1 de cada 8 niños y adolescentes entre las edades de 6 a 19 años ya tienen algún nivel de pérdida de audición" (15).

\section{Hipoacusia sensorioneural profunda (HNP)}

Este término hace referencia a una pérdida auditiva de más de $90 \mathrm{~dB} H \mathrm{HL}$ y también se lo conoce como sordera profunda. Esto puede considerarse por dos razones: la primera por perdida sensorial directamente en el oído interno y que causa daño sobre las células ciliadas ubicadas en el órgano de Corti, y la segunda por daño en el nervio auditivo que lesionan las terminaciones nerviosas de la cóclea, desatando este padecimiento y que la afectación puede ser en un solo lado del oído, es decir, unilateral o ambos lados, bilateral $(16,17)$.

También las podemos clasificar en dos categorías: congénita que está presente desde el nacimiento y que además puede ser heredada o por una alteración durante la gestación que involucra alteraciones anatómicas del oído en distintas estructuras. Otras regiones pueden estar afectadas como la cóclea, el acueducto vestibular, el canal auditivo interno, los canales semicirculares y también existen las malformaciones mixtas (18).

La segunda puede ser adquirida y es multicausal, mencionando entre ellos los traumatismos craneoencefálicos, presbiacusia (que se refiere a la pérdida de audición por la vejez), síndrome de Meniere, meningitis, medicamentos ototoxicos, exposición laboral o social a niveles altos de ruido como ser: maquinaria industrial, armas de fuego, centros recreativos, entre otros (19).

Entendiendo que el problema está en el oído interno, los síntomas que puede presentar la persona son: la dificultad que tiene para separar el habla del ruido externo además de tener problemas para oír los sonidos de alta frecuencia y sobre todo tiene mucha necesidad de que le repitan lo que se le dijo $(20,21)$.

El tratamiento se da a través de la adaptación de audífonos o por medio del implante 
coclear, esta decisión dependerá de los estudios y consideraciones audiológicas correspondientes (22).

\section{Implante coclear}

Se lleva realizando desde 1957, es un pequeño dispositivo electrónico transductor que es insertado por medio de una cirugía. Tiene como finalidad captar las ondas acústicas del medio externo y transformarlas en señales eléctricas, que son las que estimulan al nervio auditivo y a través de él, son transportadas a los centros auditivos superiores en el cerebro (23).

Este dispositivo consta de componentes internos que están ubicados en huesos y cavidades craneanas; y componentes externos, situados sobre la región temporal del cráneo. Los componentes externos a simple vista son: Micrófono, procesador del habla, antena transmisora. Y los internos insertados por medio de la cirugía son: Receptor - estimulador, Electrodos (24).

Sin duda, los avances en la tecnología son de mucha importancia, porque permiten que hoy en día estén disponibles implantes cocleares multicanales con gran capacidad de procesar la información acústica de modo más natural. De manera que, existen diferentes formas de programación, tipos de electrodos, tipos de procesadores y estrategias de preprocesamiento de la señal que se adaptan a las necesidades de cada usuario (25).

Para poder realizar el proceso de implantación hay requisitos que deben cumplirse: Se recomienda este dispositivo en personas con hipoacusia sensorioneural uni o bilateral profunda de origen coclear, con el nervio auditivo funcional. La edad no se considera un elemento de selección, por ejemplo, hace 10 años era contraindicado realizar este proceso en niños menores a 2 años, pero hoy en día se sabe que generan un resultado sorprendente en la percepción auditiva, así como el en desarrollo del lenguaje. Tomar en cuenta la edad de adquisición de las habilidades del lenguaje en el paciente, si son prelocutivos o postlocutivos para un mejor acondicionamiento. Los cuadros de otitis media crónica, deben ser tratados inicialmente antes del implante para evitar la contaminación del área. En caso de malformaciones bilaterales, por ejemplo, agenesia coclear o vía del nervio auditivo, el implante coclear está contraindicado. No presentar enfermedades psiquiátricas severas o enfermedades generales que sean contraproducentes para esta cirugía (26-28).

Criterios audiológicos de selección Estos se realizan por medio de distintos estudios:

Audiometría Tonal, prueba clínica subjetiva, que consiste en evaluar el funcionamiento del sistema auditivo. Permite hallar umbrales de audición y determina si hay pérdida auditiva (29).

Logoaudiometria, evalúa la posible alteración del procesamiento auditivo central que es la capacidad de comprensión del lenguaje hablado, es decir, la destreza que tiene el usuario para comprender y discriminar las palabras (30).

Audiometría a campo libre, prueba audio métrica esencial para la evaluación protésica, realizada dentro de una cabina sonoamortiguada. Permite evaluar la audición binaural, ganancia funcional de los audífonos (31). 
Impedanciometría, batería de pruebas que evalúan las estructuras del oído medio. Mide la impedancia acústica, los umbrales de los reflejos del tímpano, los huesecillos y la resistencia que generan los estímulos sonoros (32).

Potenciales evocados auditivos de tronco cerebral, examen similar a la electroencefalografía, que constituyen las variaciones de voltaje que se producen en una vía nerviosa sensitiva ya sea, antes, tras o durante una estimulación acústica extrínseca (33).

En algunos casos se solicita tomografía computarizada de oídos o resonancia nuclear magnética con el fin de asegurar la indemnidad de la cóclea (34).

Implantación en pacientes prelocutivos con HNP. La palabra prelocutivo hace referencia a que la sordera es de nacimiento o se dio durante los primeros años de vida, y por lo tanto no se adquirió el lenguaje de manera natural.

Niños de 0 a 3 años: En esta edad la plasticidad cerebral es similar a los que se producen naturalmente en normoyentes (respecto al desarrollo auditivo y lingüístico).

Niños entre 3 y 6 años: Muchos estudios demostraron que niños con implantación en edades precoces denotan resultados mayores en comparación con niños de edad más avanzada, debido a que la plasticidad cerebral disminuye paulatinamente. Particularmente en las edades mencionadas el niño está en pleno desarrollo auditivo y a nivel lingüístico, lo que permite una mayor estimulación activa en la corteza auditiva y así procurar desarrollar buena comunicación oral.

Adolescentes y adultos: Los candidatos que puedan reunir las condiciones para un implante en estas edades son pocos, representan un porcentaje bajo y los resultados satisfactorios logrados también son reducidos en esta población (35).

Implantación en pacientes postlocutivos con HNP. El término postlocutivo quiere decir que la pérdida auditiva se dio posterior al aprendizaje del lenguaje.

El pronóstico de rehabilitación en estos pacientes es bastante favorable, sobre todo antes de los 60 años. Si la hipoacusia fue menor o igual a 2 años, posterior al implante hay un significativo avanza en habilidades sofisticadas como oír música, entender a los interlocutores, etc. Mientras menor sea el tiempo de hipoacusia mejores resultados se tendrán.

En el caso de hipoacusia profunda por factores asociados, su pronóstico y evolución dependerá completamente de cada caso de manera particular, pero en gran parte el éxito de la habilitación o rehabilitación en esta área dependerá de los profesionales experimentados y calificados para su ejecución (36).

\section{Rehabilitación auditiva con métodos tradicionales}

Este es el proceso posterior a la implantación, los especialistas indican que aquí comienza "el trabajo de verdad". La rehabilitación, cuyo fin es que el usuario saque mayor éxito y aprovechamiento de su dispositivo. La corteza cerebral requiere entrenamiento desde lo más sencillo a lo más complejo que dependerá de la individualidad de cada caso (37). Dentro estos métodos tenemos:

Terapia auditivo verbal (TAV): Tuvo sus orígenes en Viena a principios del siglo XX. Son estrategias y técnicas utilizadas en niños, con el fin de habilitar la escucha y entender el lenguaje. Lo que se busca con esta terapia es que el niño pueda escucharse 
a sí mismo, a sus interlocutores (mediante el lenguaje hablado) y al ambiente que lo rodea. Esto basándonos en las necesidades que tenga en sus distintos niveles: psicológico, social y educativo; requiere también el apoyo indispensable de los padres para esta intervención. Dentro sus estrategias de intervención tenemos lo siguiente: Realce acústico, Acercamiento auditivo, Parafraseo, preguntar, expansión, sándwich auditivo y condicionamiento auditivo. El fin de este tipo de terapia es mejorar las habilidades auditivas y lingüísticas del usuario del implante, con una participación activa de la familia (38).

Método Verbo tonal: Este método es una combinación de emisiones orales, ritmo y entonación y elementos de comunicación por medio de movimientos corporales, vibraciones táctiles, expresividad y afectividad. Entonces se trabaja de manera individual o también en sesiones colectivas, con el fin de una mejor y mayor integración que se acerque a las necesidades y demandas de la vida real. Dentro su procedimiento general mencionamos: Orientación espacial, campo óptimo de la audición, ampliación funcional del campo óptimo de la audición, control auditivo y seguir la progresión lingüística del niño oyente (39).

Entonces este método de intervención se centra en elementos de percepción auditiva y táctil, que aprovecha los restos auditivos del paciente. Por medio de equipos modernos como ser SUVAG (Sistema Universal Verbal Auditivo de Guberina) en el cual el cuerpo humano funciona como receptor y transmisor, a través de sus elementos como los amplificadores de sonidos con vibradores, micrófonos y audífonos (40).

Lectura Labiofacial: Este tipo de terapia es ideal para pacientes postlocutivos, ya que se apoya en el componente visual por medio del movimiento de sus diferentes estructuras; labios, lengua y mandíbula. Por lo tanto, la vista se convierte en los oídos. Sin embargo, no todas las personas realizan los mismos movimientos. Por otro lado, hay muchos fonemas que tienen los mismos rasgos en su punto y modo de articulación, sin olvidar que también existen los denominados "fonemas ocultos" /g/; /x/; /k/, lo que puede generar confusiones. Al profesional que opte por el uso de este método, se recomienda que tenga un excelente entrenamiento para poder hacer uso de este recurso en combinación con otros más (41).

Lengua de signos: Por sus características está orientado a niños prelocutivos, por medio de la integración multisensorial que se puede emplear, este tipo de comunicación se realiza por gestos y por la vista. No es universal, cada país tiene su propio sistema. En la mayoría de los casos se opta por una enseñanza bilingüe (lengua de signos y lenguaje oral) (42).

Dactilología: también denominado "alfabeto manual", es un instrumento de comunicación viso-gestual que se representa por movimientos manuales (códigos dactílicos). Si bien este sistema ayuda a la identificación de la palabra, también presenta sus limitaciones. La carencia de la fonología altera el proceso de segmentación del habla, no favorece la lectura labiofacial, ya que la vista estará centrada en los dedos. También se ve alterada la sintaxis, pero en este ultimo los profesionales en esta área indican que esta dificultad se presenta en edades tempranas, pero a medida que pasa el tiempo se va adquiriendo el conocimiento de segmentación promovido por la dactilología $(43,44)$.

Comunicación bimodal: Este sistema de comunicación está dentro los métodos mix- 
tos, por lo tanto, emplea una modalidad oral-auditiva junto a la modalidad viso-gestual, de manera que tanto los signos como el habla son empleados simultáneamente para un mejor aprendizaje del lenguaje oral. Sobre el sistema que propone es sencillo de utilizar tanto para profesionales como para los padres, ya que la organización que plantea no varía. Promueve al paciente una mejor integración social, afectiva a medida de la aparición del lenguaje oral (45).

Comunicación total: Es el uso de todos los recursos de acceso a la comunicación existente (los métodos que ya hemos mencionado en este trabajo), su uso dependerá el profesional ya sea un solo método o de manera combinada (46).

\section{Rehabilitación auditiva por medio de la musicoterapia}

Es una terapia basada en la producción y audición de la música por medio de sus elementos (ritmo, sonido, melodía y armonía), los reportes mencionan en general lo beneficiosa que puede ser esta terapia en salud mental, afectiva y social, mostrando sus múltiples aplicaciones (47).

En lo que refiere a nuestro estudio de trabajo, tiene gran relevancia en la población infantil. Si bien en el apartado anterior mencionamos una gama de modelos de rehabilitación donde sus principios rigen en la detección, discriminación, identificación y comprensión de la palabra; la musicoterapia no se aleja de esta propuesta, es más, podemos decir que comparte cierta relación en ello. Veamos, respecto a la detección podríamos colocar música y que los pequeños se muevan de manera tal que al pararse el sonido ellos también lo hagan. En la discriminación podemos hacer uso de instrumentos musicales distintos y que los niños aprecien similitudes y diferencias del mismo. En la identificación sería la apreciación particular del timbre de dicho instrumento y en la comprensión un entrenamiento consistiría en escuchar una melodía y que puedan identificar por lo menos dos de los elementos que componen (48).

Los profesionales dedicados a esta área, indican que el proceso de desarrollo auditivo de un niño normoyente no es diferente de los niños con IC, solo que ellos lo aprenden unos años más tarde. Además, esta terapia promueve la creatividad en los pequeños y la improvisación por parte de los expertos será punto clave para su aplicación. Es importante mencionar que el trabajo de intervención con estos niños no solo iría de la mano de esta nueva terapia, sino que está compuesto por un grupo multidisciplinario de especialistas, como ser: psicólogos, fonoaudiólogos, audiólogos, médicos otorrinolaringólogos y terapeutas ocupacionales. Como vemos, no se desmerece la labor de ninguno de los especialistas que intervienen en el IC, pero la sugerencia de la musicoterapia es una nueva herramienta que se propone integrar sobre todo en esta población, ya que algunos estudios han denotado sus aportes satisfactorios(49).

La música en sí es una de las artes más antiguas, y es la voz humana el primer instrumento musical. Antiguamente se producía la "música" por medio de la emisión de sonidos a través de un habla prolongada y la percusión corporal. Con el paso del tiempo se crearon distintos instrumentos que emitían sonidos armoniosos, cuyas melodías tienen la capacidad de afectación en los procesos psicoanimicos, por lo tanto, tiene impacto sobre la conducta humana (50).

La palabra musicoterapia etimológicamente significa "terapia a través de la música" y su uso se emplea en distintas patologías hoy en día. Sobre nuestro tema de interés, 
es un tipo de comunicación no verbal, pero como indicamos sus elementos de sonido son de gran ayuda para este proceso de habilitación auditiva (51).

Algunos fonoaudiólogos ya hacen uso de la musicoterapia dentro la intervención y lo adjuntan a la terapia auditivo-verbal, pero por supuesto deben contar con un musicoterapeuta profesional o tener el entrenamiento necesario para su aplicación. Apoyan con ella el desarrollo neuromotor de los bebes y en cada sesión se realizan avances sobre el conocimiento de cada nueva palabra. Mejora ampliamente la sociabilidad en el niño y generan sentimientos de alegría, bienestar y relajación. Esta herramienta hace grandes aportes a la evolución del tratamiento (52).

El instrumento identificado de mejor manera por los usuarios con IC es el piano. En un estudio de 51 usuarios el $47 \%$ de todos ellos reconocieron patrones en la percepción del timbre, por medio de la constante retroalimentación. El estudio concluye en que al parecer el IC omite algunas ondas de reconocimiento de los tonos polifónicos, representando entonces esta última, como una limitación de esta terapia (53).

Hay instrumentos disponibles para evaluar estos aspectos musicales en los portadores de IC, estos son: Evaluación clínica de percepción musical de la universidad de Washington (CAMP), la prueba de apreciación musical en IC (AMICI) (54). Otra prueba es el test de percepción musical, entre otras (55).

La musicoterapia es una disciplina relativamente joven, pero con grandes expectativas. Ya que la música en sí es milenaria, la idea es sacar el mayor provecho de ella como un recurso terapéutico y continuar impulsando las investigaciones en esta área (56).

\section{Discusión y conclusiones}

En Bolivia no se encuentran artículos disponibles sobre este tema, pero en medios de comunicación de prensa escrita como los periódicos, dan a conocer datos que se realizan cirugías de implante coclear en nuestro medio. También se conoce que esta intervención quirúrgica es delicada y que el equipo médico que lo realiza está compuesto por profesionales nacionales y extranjeros.

El implante coclear en los pacientes con hipoacusia sensorioneural severa a profunda, concuerdan que permite una audición funcional. La población más beneficiada de esta tecnología son los niños, los adultos también siempre y cuando cumplan los requisitos establecidos (57).

Sobre los métodos de rehabilitación existen diversos estudios que indican que la mejor estrategia de intervención es la comunicación bimodal (58).

Otro estudio realizado en España, menciona que el método a utilizar dependerá mucho si el paciente es niño, joven o adulto, además de sus habilidades lingüísticas. Esta investigación se realizó en la población infantil indicando que la mejor intervención para ellos es la terapia auditiva-verbal (59).

La música es una combinación de sonidos ordenados con cada elemento que lo compone, además se considera que es un lenguaje universal que expresa emociones y sentimientos. Por lo tanto constituye un sistema de comunicación, así lo dan a conocer los estudiantes del conservatorio de música de Aragón España (60).

Los esfuerzos de investigación en musicoterapia han sido objeto para analizar y en- 
tender como el procesamiento de sonido se da en los usuarios con IC. En una investigación, se hizo una comparación de la percepción musical en dos dispositivos de IC que fueron probados secuencialmente, dando como resultado que no se encontraron diferencias significativas en ambos equipos; pero si se demostró que la reducción de los armónicos y los tonos puros mejoran el disfrute musical de estos usuarios. Entonces el resultado es que los pacientes implantados tienen percepción musical que, por supuesto, debe ser dado por medio de un entrenamiento a través de programas especializados (61).

Para finalizar, decimos que los hallazgos realizados en este trabajo ya se consideran evidencia científica y la finalidad es que sirvan como base para futuras investigaciones en nuestro país, pues este tipo de cirugías cada vez serán de mayor demanda y requiere el conocimiento del tema por parte de nuestros estudiantes y profesionales especialistas del área. De ese modo, no se les limitará a los usuarios a seguir únicamente métodos de rehabilitación convencionales, sino ofrecerles más estrategias de integración para aprovechar al máximo las fortalezas de cada uno de ellos y así lograr una escucha lo más normal que sea posible.

\section{Conflictos de Intereses}

Los autores declaramos no tener conflictos de interés para el presente estudio.

\section{Referencias bibliográficas}

1. Juan Pastor E. Rehabilitación en implantes cocleares. Re Med Clin Condes. 2016;27(6):834-9.

2. Manrique M, Ramos Á, de Paula Vernetta C, Gil-Carcedo E, Lassaletta L, Sanchez-Cuadrado I, et al. Guideline on cochlear implants. Acta Otorrinolaringol Esp. 2019;70(1):47-54.

3. Manrique M, Fernández S, Huarte A, Pérez N, Olleta I, García M, et al. Resultados del programa de Implantes Cocleares de la Universidad de Navarra. Rev Med Univ Navarra. 1993;38(1):21-8.

4. National Institute on Deafness and Other Communication Disorders. Implantes cocleares [Internet]. NIDCD. 2015 [citado 24 de febrero de 2020]. Disponible en: https://www.nidcd.nih.gov/es/espanol/implantes-cocleares

5. Quique B Y, Fa M. Métodos unisensoriales para la rehabilitación de la persona con implante coclear y métodos musicoterapéuticos como nueva herramienta de intervención. Rev Otorrinolaringol Cir Cabeza Cuello. 2013;73(1):94-108.

6. Bustos Rubilar M, Fuentes-López E, Castro Abarca P, Bustos Rubilar M, Fuentes-López E, Castro Abarca P. Enfoques terapéuticos en centros de intervención para niños sordos en la región metropolitana de Chile: acceso en salud y educación. Rev CEFAC. 2018;20(3):313-23.

7. Sigren V. Musicoterapia y el niño sordo. Rev Médica Clínica Las Condes. 2003;14(1):10-3.

8. Lima JP de, lervolino SMS, Schochat E. Musical and temporal auditory skills in cochlear implant users after music therapy. CoDAS. 2018;30(6):e20180006.

9. Camacho Castro AC, Fernández AH, Ferrándiz Vindel IM. Calidad de vida en niños 
pequeños portadores de un implante coclear. Aula Abierta. 2014;42(1):28-30.

10. Monsalve González A, Núñez Batalla F. La importancia del diagnóstico e intervención temprana para el desarrollo de los niños sordos: Los programas de detección precoz de la hipoacusia. Psychosoc Interv. monsalve2006;15(1):7-28.

11. Díaz C, Goycoolea M, Cardemil F. Hipoacusia: trascendencia, incidencia y prevalencia. Rev Médica Clínica Las Condes. 2016;27(6):731-9.

12. Manrique M, Valdivieso A, Ruba D, Gimeno-Vilar C, Montes-Jovellar L, Manrique R. Revisión de los criterios audiométricos en el tratamiento de la hipoacusia neurosensorial mediante audífonos y prótesis auditivas implantables. Acta Otorrinolaringológica Esp. 2008;59(1):30-8.

13. Gutiérrez CT, Merhy AS. Expectativas del implante coclear. Rev Inst Enf Resp Mex. 2001;14(3):5.

14. Gutiérrez-Farfán I, Arch-Tirado E, Lino-González AL, Jardines-Serralde LJ. Daño auditivo inducido por ruido recreativo. Salud Pública México. 2018;60(2):126-126.

15. Daniel E. Noise and hearing loss: a review. J Sch Health. 2007;77(5):225-31.

16. Peñaranda A, Mendieta JC, Perdomo JA, Aparicio ML, Marín LM, García JM, et al. Beneficios económicos del implante coclear para la hipoacusia sensorineural profunda. Rev Panam Salud Pública. 2012;31(4):325-31.

17. Cheng L-J, Soon SS, Wu DB-C, Ju H, Ng K. Cost-effectiveness analysis of bilateral cochlear implants for children with severe-to-profound sensorineural hearing loss in both ears in Singapore. PloS One. 2019;14(8):e0220439.

18. Ontario Health (Quality). Implantable Devices for Single-Sided Deafness and Conductive or Mixed Hearing Loss: A Health Technology Assessment. Ont Health Technol Assess Ser. 2020;20(1):1-165.

19. Oliveira C, Machado M, Zenha R, Azevedo L, Monteiro L, Bicho A. [Congenital or Early Acquired Deafness: An Overview of the Portuguese Situation, from Diagnosis to Follow-Up]. Acta Med Port. 2019;32(12):767-75.

20. Sennaroğlu L, Bajin MD. Classification and Current Management of Inner Ear Malformations. Balk Med J. 2017;34(5):397-411.

21. Sennaroglu L. Cochlear implantation in inner ear malformations--a review article. Cochlear Implants Int. 2010;11(1):4-41.

22. López-Sisniega JA, González-Domínguez S, Cardona-Martínez C, Paquot-Chico RO, Valdés-Oberhauser LM. Malformaciones congénitas de cóclea e implante coclear. An Orl Mex. 2019;64(3):102-11.

23. Naples JG, Ruckenstein MJ. Cochlear Implant. Otolaryngol Clin North Am. 2020;53(1):87-102.

24. Shepherd RK, McCreery DB. Basis of electrical stimulation of the cochlea and the cochlear nucleus. Adv Otorhinolaryngol. 2006;64:186-205.

25. Gordon KA, Papsin BC, Harrison RV. Toward a battery of behavioral and objective measures to achieve optimal cochlear implant stimulation levels in children. Ear Hear. 2004;25(5):447-63. 
26. Cohen NL. Cochlear implant candidacy and surgical considerations. Audiol Neurootol. 2004;9(4):197-202.

27. Sampaio ALL, Araújo MFS, Oliveira CACP. New criteria of indication and selection of patients to cochlear implant. Int J Otolaryngol. 2011;2011:573968.

28. Varadarajan VV, Sydlowski SA, Li MM, Anne S, Adunka OF. Evolving Criteria for Adult and Pediatric Cochlear Implantation. Ear Nose Throat J. 2020;145561320947258.

29. Pirodda E. Tonal audiometry. Otorinolaringol Ital. 1951;19(6):411-60.

30. De Lacerda AP. Speech audiometry. Rev Bras Oto-Rino-Laringol. 1954;22(1):96107.

31. Sortini AJ, Flake CG. Speech audiometry testing for pre-school children. The Laryngoscope. 1953;63(10):991-7.

32. Pérez-Villa YE, Mena-Ramírez ME, Chamlati Aguirre LE, Mora-Magaña I, Gutiérrez-Farfán IS. Utilidad diagnóstica del reflejo acústico para predecir audición en población pediátrica. Acta Otorrinolaringológica Esp. 2014;65(6):332-8.

33. Calero Del Castillo JB, Guillén Martínez AJ, García-Purriños García F. Search for normality criteria of auditory brain responses and auditory steady state response with free-field stimulation. Acta Otorrinolaringol Esp. 2019;70(5):258-64.

34. Ramos-Macías Á, Borkoski S, Juan Carlos FG, Miguel Á. Implante coclear. Estado actual y futuro. Rev Médica Clínica Las Condes. 2016;27:798-807.

35. Chaverri-Polini J, Chaverri-Polini J. El implante coclear: sus indicaciones. Acta Médica Costarric. 2018;60(3):132-5.

36. Manrique M, Ramos A, Morera C, Cenjor C, Lavilla MJ, Boleas MS, et al. Analysis of the cochlear implant as a treatment technique for profound hearing loss in pre and postlocutive patients. Acta Otorrinolaringol Esp. 2006;57(1):2-23.

37. Fadda S. Psychological aspects when counseling families who have children with cochlear implants. J Matern-Fetal Neonatal Med Off J Eur Assoc Perinat Med Fed Asia Ocean Perinat Soc Int Soc Perinat Obstet. 2011;24 Suppl 1:104-6.

38. Ivone FN, Schochat E. Auditory processing maturation in children with and without learning difficulties. -Fono Rev Atualizacao Cient. 2005;17(3):311-20.

39. Motta G, lengo M, Tranchino G, Motta G, D'Angelo L, Salafia M, et al. Speech rehabilitation of children with severe and profound deafness using the verbo-tonal method. Acta Otorhinolaryngol Ital Organo Uff Della Soc Ital Otorinolaringol E Chir Cerv-facc. 1987;7(15):1-15.

40. Cortés M del CM, Morón MTP, Góngora DP, López-Liria R, Acién FL. Métodos De Intervención En Discapacidad Auditiva. Int J Dev Educ Psychol. 2008;3(1):21924.

41. Gasso T, Gasso JM. Rehabilitation by lip-reading \& auditory training of post-dihydrostreptomycin deafness; its special characteristics. Acta Otorinolaryngol Iber Am. 1957;8(2):289-97.

42. Gámez MCGG, Cáceres RG. Uso de la Lengua de Signos Española en la educa- 
ción del alumnado sordo. Eticnet Rev Científica Electrónica Educ Comun En Soc Conoc. 2012;12(2):231-58.

43. Kunkler JG. Typing, the natural language of the deaf and dumb. Infirm. 1948;6(11):354-8.

44. Herrera V, Puente A, Alvarado JM, Ardila A. códigos de lectura en sordos: la dactilología y otras estrategias visuales y kinestésicas. Rev Latinoam Psicol. 2007;39(2):269-86.

45. Perez I, Velasco C. Sistemas y recursos de apoyo a la comunicación y al lenguaje de los alumnos sordos. Revista Latinoamericana de Inclusión Educativa. 2009;3(1):77-93.

46. Tobey EA, Shin S, Prashant MS, Geers A. Spoken Word Recognition in Adolescent Cochlear Implant Users During Quiet and Multi-Speaker Babble Conditions. Otol Neurotol Off Publ Am Otol Soc Am Neurotol Soc Eur Acad Otol Neurotol. 2011;32(3):413-8.

47. Contreras Usseglio RM. Musicoterapia. Rev Actual Clínica Investiga. 2011;9(1):447.

48. Miranda MC, Hazard SO, Miranda PV, Miranda MC, Hazard SO, Miranda PV. La música como una herramienta terapéutica en medicina. Rev Chil Neuro-Psiquiatr. 2017;55(4):266-77.

49. Plaza JLA. El desarrollo creativo en Educación Musical: del genio artístico al trabajo colaborativo. Educação. 2012;37(1):31-44.

50. Mercadal M, Martí P. Music therapy. Rev Enfermeria Barc Spain. 2005;28(3):548.

51. Sanz JIP. El Concepto De Musicoterapia a Través De La Historia. Rev Interuniv Form Profr. 2001;(42):19-31.

52. Oliveira MF de, Oselame GB, Neves EB, Oliveira EM de. musicoterapia como ferramenta terapêutica no setor da saúde: uma revisão sistemática. Rev Universidade Vale Rio Verde. 2014;12(2):871-9.

53. Donnelly PJ, Guo BZ, Limb CJ. Perceptual fusion of polyphonic pitch in cochlear implant users. J Acoust Soc Am. 2009;126(5):128-33.

54. Kang R, Nimmons GL, Drennan W, Longnion J, Ruffin C, Nie K, et al. Development and validation of the University of Washington Clinical Assessment of Music Perception test. Ear Hear. 2009;30(4):411-8.

55. Nimmons GL, Kang RS, Drennan WR, Longnion J, Ruffin C, Worman T, et al. Clinical assessment of music perception in cochlear implant listeners. Otol Neurotol Off Publ Am Otol Soc Am Neurotol Soc Eur Acad Otol Neurotol. 2008;29(2):14955.

56. Kong Y-Y, Cruz R, Jones JA, Zeng F-G. Music perception with temporal cues in acoustic and electric hearing. Ear Hear. 2004;25(2):173-85.

57. Hoppe U, Liebscher T, Hornung J. Cochlear implant fitting strategies. HNO. 2017;65(7):546-51. 
58. Seeber BU, Baumann U, Fastl H. Localization ability with bimodal hearing aids and bilateral cochlear implants. J Acoust Soc Am. 2004;116(3):1698-709.

59. Díaz-Uña A, Benito-González F, Gorospe-Arocena JM. Resultados de la implantación coclear en edad pediátrica. Estudio retrospectivo en 72 pacientes. Rev ORL. 2019;10(1):35-44.

60. Muñoz ST. Calidad de vida de los estudiantes de música del Conservatorio de Música de Aragón. Rev Electrónica LEEME. 2009;(24):5.

61. Foteff C, Kennedy S, Milton AH, Deger M, Payk F, Sanderson G. Cost-Utility Analysis of Cochlear Implantation in Australian Adults. Otol Neurotol Off Publ Am Otol Soc Am Neurotol Soc Eur Acad Otol Neurotol. 37(5):454-61. 